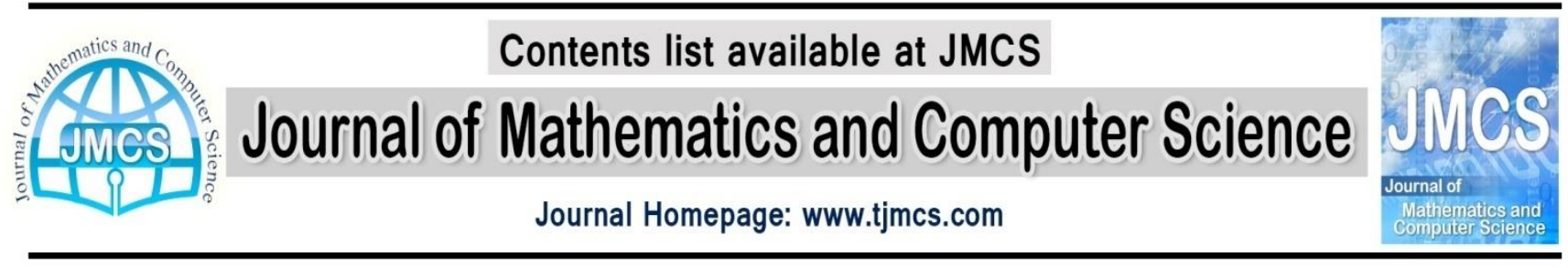

\title{
Short-Term Electricity Price Forecasting Using Optimal TSK Fuzzy Systems
}

\author{
Saeid Eslahi Tatafi ${ }^{\mathrm{a}}$, Gholam Ali Heydari ${ }^{\mathrm{b}}$, Ali Akbar Gharaveisi ${ }^{\mathrm{c}}$ \\ ${ }^{a}$ Department of Electrical Engineering, Sowmesara branch, Islamic Azad University, Sowmesara, Iran \\ ${ }^{b}$ Department of Mathematics, Shahid Bahonar University of Kerman, Kerman, Iran \\ ${ }^{c}$ Department of Electrical Engineering, Shahid Bahonar University of Kerman, Kerman, Iran \\ Article history: \\ saeideslahi.ce@gmail.com \\ Received August 2014 \\ Accepted September 2014 \\ Available online October 2014
}

\begin{abstract}
Since all financial transactions in restructured power markets are based on electricity prices, it is necessary that the price of electric power be predicted precisely. Some particular features such as: nonlinearity, non-stationary behaviors, as well as volatility of electricity prices make such a prediction a very challenging task. In this paper, a new structure of TSK fuzzy systems is presented that provides high order TSK fuzzy systems from lower orders which have capability of modeling and forecasting chaotic time series. The method used for optimization of fuzzy systems is the Interior point method. Using this method for forecasting electricity price is useful because of its chaotic behavior. The results are compared with RBF neural network and TSK fuzzy system presents better results.
\end{abstract}

Keywords: Forecasting; TSK fuzzy systems; Interior Point Method; RBF neural network.

\section{Introduction}

One of the most essential issues in restructured electric power market to handle all kind of energy transaction (i.e. short-term, midterm and long-term transaction) is price forecasting [1,2,3,4,5]. Various kinds of electricity price prediction methods are being used for different transactions schemes. Day-ahead price forecasting is one of the most important scheme with a crucial importance especially in pool-based power markets [6,7]. During the last two decades, many researchers have focused on the field of price prediction using different approaches that are reviewed in the following: Hsiao suggested an artificial neural network technique to predict long-term electricity price for European Energy Exchange. In his study, autocorrelation function is used for data analysis, while a neural network with $\{5: 7: 2\}$ structure is exploited to predict the price in the scheme of one, two, three days and a week as well as three months ahead which performs an automatic regression approach with 
respect to prediction error [3]. In [8] a fuzzy-neural network is employed for price forecasting in Spain energy market that results more accurate prediction in comparison with ARMA and Wavelet_ARIMA. Automatic Dynamic Harmonic Regression is applied to a day-ahead price prediction of Spain energy market in which the reported results show a better performs than ARIMA [7]. In 2005, WaveletARIMA is used in another study for price prediction in Spain energy market. In the first step of WARIMA, historical data of the price pertaining to the previous day is classified into four series. In the second step, an ARMA model for each of the four series is designed to predict 24 values for the day ahead. In the last step, the predicted values by ARIMA are combined using inverse wavelet transform, and final price prediction for the day ahead is obtained [6]. Antonio proposed different techniques such as Transfer Function, and Dynamic Regression for price prediction [7]. ARIMA, Neural Networks, and Wavelet are employed to predict electricity price in PJM market in year 2002 [6]. The results of this study show a better performance of time series techniques in comparison with wavelet and neural networks. The results also show that DR and TF, among the other time series techniques, are more effective than ARIMA models. WL models also behave similar to ARIMA models [1]. Reinaldo \& Javier employed GARCH (Generalized Auto Regressive Conditional Heteroskedastic) to predict electricity price in Spain and California markets and showed that GARCH results are more reliable than those of ARIMA. Especially, when electrical load data are included in GARCH model the results may be improved [1]. Neuro-Fuzzy models were employed in a study to predict Locational Marginal Prices. Since LMPs in one region are increased due to power interruption and congestion, linguistic and numerical information are used for prediction and fuzzy logic is therefore employed to transform linguistic data into numerical [5]. A significant feature of hourly electricity price which challenges the prediction process is the high frequency of hourly price signal(see figure 1) [1,9] which demands for the use of TSK fuzzy systems as powerful tools to handle such nonlinear signals. A studywas done on the short term forecasting of electricity price using neural network (FPNN) in 2010,the results demonstrated of this method over the probabilistic neural network(PNN) [14].

In this paper various order TSK fuzzy systems have been employed to forecast day ahead electricity price according to PJM market data. Tuning of these TSK fuzzy systems is done by using the Interior Point Method such that the proposed system gives the right output for an specific input. Results show the advantage of TSK fuzzy systems in forecasting electricity price in comparison with RBF neural network. However it is also shown that zero order TSK fuzzy system has the best performance among other TSK systems.

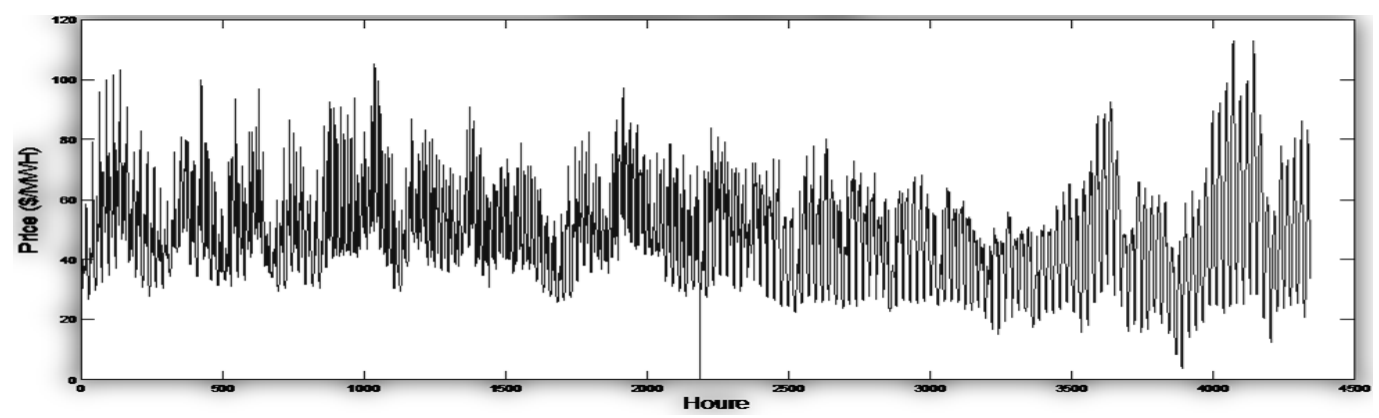

Fig.1: Typical electricity price variations 


\section{Proposed TSK Fuzzy Systems}

A typical fuzzy system consists of four fundamental parts [10]: 1- Fuzzifer, 2-Rule Base, 3- Inference Engine, 4- Defuzzifer As shown above, the input (which is the vector [x1 x2:::xm]T ) is converted from crisp to fuzzy which is done comparing input value by input membership functions, the result then is employed by inference engine which generates a fuzzy output according to input value and fuzzy rules. finally a real world signal y is produced by converting fuzzy output back to a crisp value. The important specification of a TSK fuzzy system, is the way it produces output signal. The most popular types of TSK fuzzy systems are: 1- Zero Order (or Constant) and 2- First Order (or Linear) [10]. The 1-th rule of a typical rule base of a Zero order TSK system and a First Order TSK system are as noted bellow respectively:

$$
\begin{aligned}
& \text { If } x_{1} \text { is } A_{1}^{l} \& x_{2} \text { is } A_{2}^{l} \& \ldots . \& x_{m} A_{m}^{l} \text { Then } y=a_{0}^{l} \\
& \text { If } x_{1} \text { is } A_{1}^{l} \& x_{2} \text { is } A_{2}^{l} \& \ldots . \& x_{m} A_{m}^{l} \text { Then } y=a_{0}^{l}+a_{1}^{l} x_{1}+a_{2}^{l} x_{2}+\ldots .+a_{m}^{l} x_{m}
\end{aligned}
$$

Where $A_{i}^{l}$ is a membership function of i-th input, $a^{l}$ is the constant value of 1-th rule output and $a^{l}$ is the coefficient of $\mathrm{i}$-th input variable in the linear combination forming output of the 1-th rule $y^{l}$. The total effect of rules is usually calculated by weighted averaging:

$$
y=\frac{\sum_{l=1}^{r} w^{l} y^{l}}{\sum_{l=1}^{r} w^{l}}
$$

Where $\mathrm{r}$ is total number of rules and $w^{l}$ is the weight assigned to 1-th rule, which shows how much each rule effects on the output and is calculated according to the dependency of input variables (x1...xm) to membership functions of 1-th rule $\left(A_{1}^{l} \ldots A_{m}^{l}\right)$ [10]. As it is clear the operation of defuzzifier is almost done by the inference engine and output calculation is much simpler than Mamdani fuzzy system.

\section{High Order TSK}

The idea of higher order TSK fuzzy systems was first introduced by Buckley [11]. Supposing the I -th rule of a typical TSK fuzzy system from order $n$ with $m$ inputs:

$$
\text { If } x_{1} \text { is } A_{1}^{l} \& x_{2} \text { is } A_{2}^{l} \& \ldots . \& x_{m} A_{m}^{l} \text { Then } y=\sum_{\substack{j_{1}+\ldots+j_{m} \leq n \\ j_{1}, j_{2}, \ldots, j_{m} \geq 0}}\left(a_{j_{1}, j_{2}, \ldots, j_{m}}^{l}\right) x_{1}^{j_{1}} x_{1}^{j_{2}} \ldots x_{m}^{j_{m}}
$$

\section{Electricity Price Forecasting Using Proposed TSK Fuzzy Systems}

In this section TSK fuzzy system, introduced in chapter 3, is used in order to forecast electricity price data in pjm market. in general, there are two methods used in forecasting one-day ahead electricity price. 
In this section, the direct method is used to forecast one-day ahead electricity price. That is, using 24 fuzzy models in forecasting 24-hour ahead. The Input data used in training fuzzy system is presented bellow:

$$
\left\{\begin{array}{l}
X=\left[P_{h-24}, P_{h-48}, P_{h-72}, P_{h-96},\right. \\
\left.\quad P_{h-120}, P_{h-144}, P_{h-168}, P_{h-192}, \ldots, P_{h-N^{* 24}}\right] \\
y=P_{h}
\end{array}\right.
$$

For example, if $\mathrm{N}=5$,to predict the price of electricity on 24 May 2006, at 1 a.m, Data from 5 days ago at the same time is used. Four days are considered as the input and the day before the forecasting day as the output of the fuzzy system. After the training phase; for predicting the $\mathrm{P}(\mathrm{h}+24)$, $\mathrm{P}(\mathrm{h})$ is also considered as the input (figure 2). The Membership functions of the input are selected according to figure 3 .

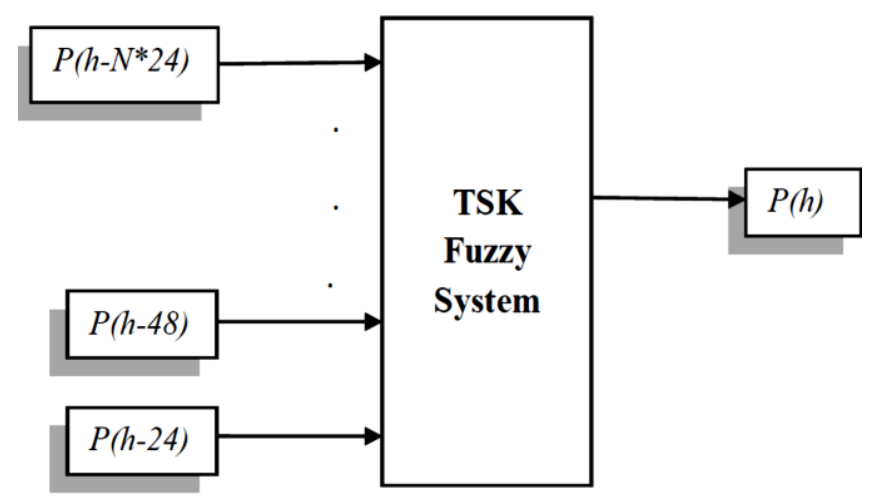

Figure.2: inputs of the fuzzy system

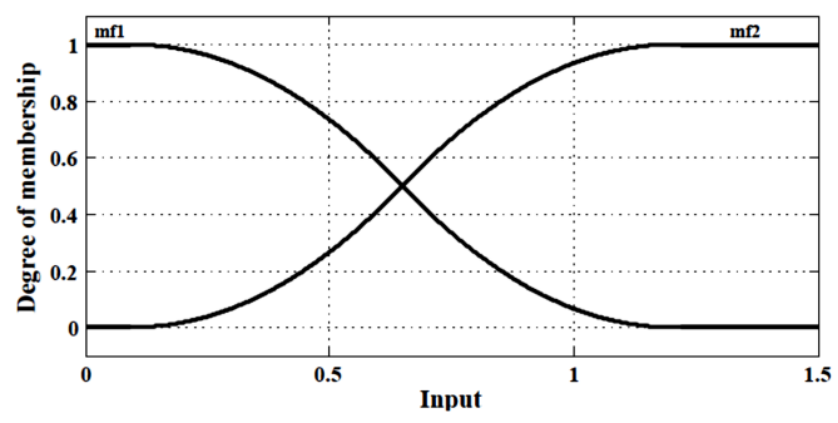

Figure.3: Input Membership function

The interior point method is used to tune the parameters of fuzzy systems [12]. Predicted results for the four weeks of winter, spring, summer and autumn of 2006 are shown in Table 1 to show the Performance of fuzzy system in forecasting, two error criteria of RMSE and MAPE are used. 
Table 1. Results for the four weeks of winter, spring, summer and autumn of 2006

\begin{tabular}{|c|c|c|c|c|c|c|c|}
\hline \multirow{3}{*}{ Day } & \multicolumn{5}{|c|}{ Winter Week : March 14, 2006 to March 19, 2006} & \multirow{2}{*}{\multicolumn{2}{|c|}{ Second-Order-TSK }} \\
\hline & & \multicolumn{2}{|c|}{ Zero-Order-TSK } & \multicolumn{2}{|c|}{ First-Order-TSK } & & \\
\hline & $\begin{array}{l}N=\text { number } \\
\text { of train data }\end{array}$ & RMSE & MAPE & RMSE & MAPE & RMSE & MAPE \\
\hline Monday & 5 & 3.4694 & 5.9107 & 6.6923 & 13.122 & 10.471 & 14.673 \\
\hline Tuesday & 5 & 8.9541 & 12.616 & 8.9591 & 14.896 & 13.930 & 17.906 \\
\hline Wednesday & 5 & 4.0447 & 6.3778 & 8.9604 & 12.041 & 11.963 & 16.093 \\
\hline Thursday & 5 & 4.3196 & 7.3617 & 10.812 & 18.739 & 14.621 & 20.785 \\
\hline Friday & 5 & 6.1919 & 10.199 & 7.7608 & 9.8222 & 9.7510 & 12.956 \\
\hline Saturday & 5 & 6.0479 & 9.5732 & 9.9936 & 17.697 & 12.851 & 18.541 \\
\hline Sunday & 10 & 8.4940 & 12.5453 & 29.460 & 47.805 & 24.961 & 43.760 \\
\hline
\end{tabular}

\begin{tabular}{|c|c|c|c|c|c|c|c|}
\hline \multirow{3}{*}{ Day } & \multicolumn{7}{|c|}{ spring Week : May 14, 2006 to May 19, 2006} \\
\hline & & \multicolumn{2}{|c|}{ Zero-Order-TSK } & \multicolumn{2}{|c|}{ First-Order-TSK } & \multicolumn{2}{|c|}{ Second-Order-TSK } \\
\hline & $\begin{array}{l}\mathrm{N}=\text { number } \\
\text { of train data }\end{array}$ & RMSE & MAPE & RMSE & MAPE & RMSE & MAPE \\
\hline Monday & 10 & 2.0422 & 5.1259 & 4.4937 & 8.5300 & 39.743 & 71.990 \\
\hline Tuesday & 5 & 1.7249 & 4.3821 & 2.7596 & 6.3418 & 11.275 & 21.696 \\
\hline Wednesday & 5 & 5.3715 & 7.6428 & 4.9327 & 7.5700 & 5.9805 & 8.6192 \\
\hline Thursday & 5 & 2.4705 & 4.6640 & 1.9365 & 3.9200 & 14.312 & 23.456 \\
\hline Friday & 5 & 2.9729 & 4.7301 & 3.8075 & 6.2792 & 28.471 & 51.202 \\
\hline Saturday & 10 & 4.4905 & 9.0168 & 7.2440 & 16.990 & 16.648 & 37.676 \\
\hline Sunday & 5 & 4.8952 & 11.788 & 3.9345 & 9.3731 & 8.557 & $\mathbf{1 4 . 1 0 5}$ \\
\hline
\end{tabular}

\begin{tabular}{|c|c|c|c|c|c|c|c|}
\hline \multicolumn{7}{|c|}{ Summer Week : August 21, 2006 to August 27, 2006 } \\
\hline \multirow{2}{*}{ Day } & \multicolumn{2}{|c|}{ Zero-Order-TSK } & \multicolumn{2}{c|}{ First-Order-TSK } & \multicolumn{2}{c|}{ Second-Order-TSK } \\
\cline { 2 - 8 } & $\begin{array}{c}\text { N=number } \\
\text { of train data }\end{array}$ & RMSE & MAPE & RMSE & MAPE & RMSE & MAPE \\
\hline Monday & $\mathbf{1 0}$ & $\mathbf{6 . 4 3 1 0}$ & $\mathbf{6 . 9 5 6 3}$ & $\mathbf{1 0 . 9 8 4}$ & $\mathbf{1 7 . 3 0 9}$ & $\mathbf{1 4 . 0 6 3}$ & $\mathbf{2 4 . 4 7 9}$ \\
\hline Tuesday & $\mathbf{5}$ & $\mathbf{1 . 8 3 0 9}$ & $\mathbf{2 . 3 8 3 5}$ & $\mathbf{3 . 6 6 4 6}$ & $\mathbf{6 . 7 9 6 3}$ & $\mathbf{1 1 . 7 8 7}$ & $\mathbf{1 9 . 8 9 9}$ \\
\hline Wednesday & 5 & $\mathbf{2 . 5 5 4 1}$ & $\mathbf{4 . 2 1 7 1}$ & $\mathbf{3 . 3 7 6 6}$ & $\mathbf{5 . 1 4 1 5}$ & $\mathbf{9 . 4 4 6 7}$ & $\mathbf{1 0 . 5 4 7}$ \\
\hline Thursday & $\mathbf{5}$ & $\mathbf{2 . 6 0 6 9}$ & $\mathbf{4 . 1 1 9 0}$ & $\mathbf{3 . 0 9 6 5}$ & $\mathbf{4 . 6 9 1 3}$ & $\mathbf{1 0 . 7 6 4}$ & $\mathbf{1 4 . 3 7 0}$ \\
\hline Friday & 5 & 4.9900 & $\mathbf{5 . 7 0 5 4}$ & $\mathbf{4 . 0 6 2 8}$ & $\mathbf{5 . 8 3 9 8}$ & $\mathbf{1 2 . 4 1 7}$ & $\mathbf{1 2 . 9 5 0}$ \\
\hline Saturday & $\mathbf{5}$ & $\mathbf{4 . 5 7 2 0}$ & $\mathbf{8 . 1 7 4 3}$ & $\mathbf{4 . 9 7 1 7}$ & $\mathbf{8 . 4 8 5 2}$ & $\mathbf{8 . 8 7 6 7}$ & $\mathbf{1 1 . 9 4 0}$ \\
\hline Sunday & 10 & $\mathbf{5 . 6 6 0 0}$ & $\mathbf{1 0 . 2 2 2}$ & $\mathbf{2 1 . 2 0 4}$ & $\mathbf{2 9 . 6 0 4}$ & $\mathbf{2 2 . 8 6 5}$ & $\mathbf{3 1 . 8 6 0}$ \\
\hline
\end{tabular}

\begin{tabular}{|c|c|c|c|c|c|c|c|}
\hline \multicolumn{7}{|c|}{ Fall Week : November 13, 2006 to November 19, 2006 } \\
\hline \multirow{2}{*}{ Day } & \multicolumn{2}{|c|}{ Zero-Order-TSK } & \multicolumn{2}{c|}{ First-Order-TSK } & \multicolumn{2}{c|}{ Second-Order-TSK } \\
\cline { 2 - 8 } & $\begin{array}{c}\text { N=number } \\
\text { of train data }\end{array}$ & RMSE & MAPE & RMSE & MAPE & RMSE & MAPE \\
\hline Monday & 10 & 7.3866 & 11.203 & 13.610 & 20.244 & 28.340 & $\mathbf{3 3 . 4 7 2}$ \\
\hline Tuesday & 5 & $\mathbf{3 . 7 7 0 0}$ & $\mathbf{5 . 3 8 3 9}$ & $\mathbf{7 . 6 1 3 7}$ & $\mathbf{1 0 . 9 2 4}$ & $\mathbf{3 0 . 5 6 1}$ & $\mathbf{4 5 . 0 1 8}$ \\
\hline Wednesday & 5 & $\mathbf{2 . 2 7 5 3}$ & $\mathbf{3 . 7 5 1 8}$ & $\mathbf{4 . 5 6 1 7}$ & $\mathbf{6 . 4 5 7 0}$ & $\mathbf{2 3 . 3 1 0}$ & $\mathbf{3 3 . 2 6 4}$ \\
\hline Thursday & 5 & 2.5290 & $\mathbf{5 . 7 4 4 5}$ & $\mathbf{8 . 0 2 2 3}$ & $\mathbf{1 2 . 2 1 9}$ & $\mathbf{4 1 . 8 1 0}$ & $\mathbf{6 0 . 5 3 2}$ \\
\hline Friday & 5 & 4.7729 & $\mathbf{9 . 0 4 4 7}$ & $\mathbf{7 . 3 2 8 8}$ & $\mathbf{1 3 . 2 7 6}$ & $\mathbf{1 3 . 8 5 0}$ & $\mathbf{2 7 . 1 7 1}$ \\
\hline Saturday & 5 & $\mathbf{8 . 2 7 0 0}$ & $\mathbf{1 6 . 2 1 3}$ & $\mathbf{1 1 . 9 7 9}$ & $\mathbf{2 3 . 0 2 4}$ & $\mathbf{1 4 . 6 0 7}$ & $\mathbf{2 8 . 4 9 0}$ \\
\hline Sunday & 5 & 5.8154 & $\mathbf{9 . 9 3 6 1}$ & $\mathbf{5 . 8 7 0 0}$ & $\mathbf{1 0 . 0 5 2}$ & $\mathbf{1 0 . 6 6 3}$ & $\mathbf{2 2 . 0 7 6}$ \\
\hline
\end{tabular}


According to the results and the Modeling Flowchart, it is concluded that the best model for short term electricity price forecasting is Zero-Order-TSK fuzzy.

RBF neural network [13] is used in forecasting and its comparison with the TSK fuzzy system. While the day before the current day (which is going to be predicted) has the most correlation with it so, eighth days that have more correlation with the day before the current day, are chosen and used as training data. The input vector used for training is defined as follows:

$$
\left\{\begin{aligned}
X= & {\left[P_{h-24}, P_{h-25}, P_{h-48}, P_{h-49}, P_{h-72}, P_{h-73}, P_{h-96}, P_{h-97},\right.} \\
& \left.\quad P_{h-120}, P_{h-121}, P_{h-144}, P_{h-145}, P_{h-168}, P_{h-169}, P_{h-192}, P_{h-193}\right] \\
y= & P_{h}
\end{aligned}\right.
$$

In this section two days of year 2006 have been selected for instance to be forecasted. Results of day ahead forecast made by fuzzy system and RBF neural network are illustrated on figure 4.
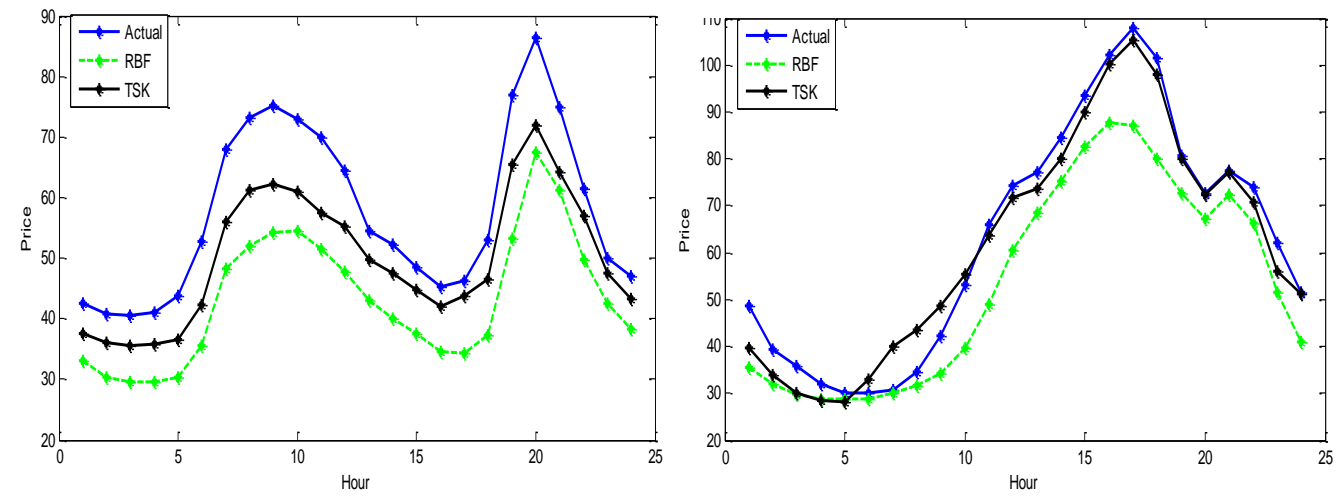

Figure 4. Results of day ahead forecast for 20 march 2006 and 27 may 2006

Now results of forecast for four weeks (selected form winter, spring, summer and fall respectively) made by fuzzy system and RBF neural network, are given in table 2 .

Table 2. Results of forecast for four weeks (selected form winter, spring, summer and fall respectively)

\begin{tabular}{|l|c|c|c|c|c|c|}
\hline \multicolumn{7}{|c|}{ Winter Week : March 14, 2006 to March 19, 2006 } \\
\hline \multirow{2}{*}{ Day } & \multicolumn{3}{|c|}{ TSK } & \multicolumn{3}{c|}{ RBF } \\
\cline { 2 - 7 } & $\begin{array}{c}\text { N=number } \\
\text { of train data }\end{array}$ & RMSE & MAPE & $\begin{array}{c}\text { N=number } \\
\text { of train data }\end{array}$ & RMSE & MAPE \\
\hline Monday & 10 & 3.4694 & 5.9107 & 17 & 4.4226 & 6.4489 \\
\hline Tuesday & 5 & 8.9541 & 12.616 & 17 & 7.2725 & 10.141 \\
\hline Wednesday & 5 & 4.0447 & 6.3778 & 17 & 6.9525 & 9.4468 \\
\hline Thursday & 10 & 4.3196 & 7.3617 & 17 & 2.2073 & 3.2917 \\
\hline Friday & 5 & 6.1919 & 10.199 & 17 & 8.2520 & 16.260 \\
\hline Saturday & 5 & 6.0479 & 9.5732 & 17 & 7.3135 & 12.863 \\
\hline Sunday & 10 & 8.4940 & 12.5453 & 17 & 15.105 & 24.999 \\
\hline
\end{tabular}




\begin{tabular}{|l|c|c|c|c|c|c|}
\hline \multicolumn{7}{|c|}{ spring Week : May 14, 2006 to May 19, 2006 } \\
\hline \multirow{2}{*}{ Day } & \multicolumn{3}{|c|}{ TSK } & \multicolumn{3}{c|}{ RBF } \\
\cline { 2 - 7 } & $\begin{array}{c}\text { N=number } \\
\text { of train data }\end{array}$ & RMSE & MAPE & $\begin{array}{c}\text { N=number } \\
\text { of train data }\end{array}$ & RMSE & MAPE \\
\hline Monday & 10 & 2.0422 & 5.1259 & 17 & 4.5725 & 9.1932 \\
\hline Tuesday & 5 & 1.7249 & 4.3821 & 17 & 2.3400 & 5.0720 \\
\hline Wednesday & 5 & 5.3715 & 7.6428 & 17 & 4.4795 & 7.5081 \\
\hline Thursday & 5 & 2.4705 & 4.6640 & 17 & 3.2597 & 5.2700 \\
\hline Friday & 5 & 2.9729 & 4.7301 & 17 & 3.8250 & $\mathbf{6 . 9 8 2 2}$ \\
\hline Saturday & 10 & 4.4905 & $\mathbf{9 . 0 1 6 8}$ & 17 & 7.5669 & 7.5669 \\
\hline Sunday & 5 & 4.8952 & 11.788 & 17 & 3.9910 & 11.009 \\
\hline
\end{tabular}

\begin{tabular}{|c|c|c|c|c|c|c|}
\hline \multicolumn{7}{|c|}{ Summer Week : May 21, 2006 to May 27, 2006} \\
\hline \multirow{2}{*}{ Day } & \multicolumn{3}{|l|}{ TSK } & \multicolumn{3}{|l|}{ RBF } \\
\hline & $\begin{array}{l}N=\text { number } \\
\text { of train data }\end{array}$ & RMSE & MAPE & $\begin{array}{l}\mathrm{N}=\text { number } \\
\text { of train data }\end{array}$ & RMSE & MAPE \\
\hline Monday & 10 & 6.4310 & 6.9563 & 17 & 8.0660 & 14.613 \\
\hline Tuesday & 5 & 1.8309 & 2.3835 & 17 & 4.6889 & 6.8710 \\
\hline Wednesday & 5 & 2.5541 & 4.2171 & 17 & 3.9966 & 6.7615 \\
\hline Thursday & 5 & 2.6069 & 4.1190 & 17 & 2.2040 & 3.1946 \\
\hline Friday & 5 & 4.9900 & 5.7054 & 17 & 3.6820 & 4.4105 \\
\hline Saturday & 5 & 4.5720 & 8.1743 & 17 & 10.769 & 14.134 \\
\hline Sunday & 10 & 5.6600 & 10.222 & 17 & 6.4744 & 10.798 \\
\hline
\end{tabular}

\begin{tabular}{|c|c|c|c|c|c|c|}
\hline \multicolumn{7}{|c|}{ Fall Week : November 13, 2006 to November 19, 2006} \\
\hline \multirow{2}{*}{ Day } & \multicolumn{3}{|l|}{ TSK } & \multicolumn{3}{|l|}{ RBF } \\
\hline & $\begin{array}{l}\mathrm{N}=\text { number } \\
\text { of train data }\end{array}$ & RMSE & MAPE & $\begin{array}{l}\mathrm{N}=\text { number } \\
\text { of train data }\end{array}$ & RMSE & MAPE \\
\hline Monday & 10 & 7.3866 & 10.203 & 17 & 4.2100 & 10.961 \\
\hline Tuesday & 5 & 3.7700 & 5.3839 & 17 & 6.9772 & 10.175 \\
\hline Wednesday & 5 & 2.2753 & 3.7518 & 17 & 4.0846 & 5.5591 \\
\hline Thursday & 5 & 2.5290 & 5.7445 & 17 & 2.9324 & 5.9996 \\
\hline Friday & 5 & 4.7729 & 9.0447 & 17 & 3.9841 & 6.3811 \\
\hline Saturday & 10 & 8.2700 & 16.213 & 17 & 5.5070 & 12.740 \\
\hline Sunday & 5 & 5.8154 & 9.9361 & 17 & 9.8239 & 20.186 \\
\hline
\end{tabular}

Table 3.

PJM Interconnection: statistical measures for seasons of 2006

\begin{tabular}{lcccc}
\hline Week & \multicolumn{2}{c}{ TSK } & \multicolumn{2}{c}{ RBF } \\
& RMSE & MAPE & RMSE & MAPE \\
Winter & $\mathbf{5 . 9 3 1 6}$ & $\mathbf{9 . 5 5 1 9}$ & 7.3607 & 11.921 \\
Spring & $\mathbf{3 . 4 2 4 6}$ & $\mathbf{6 . 5 9 0 6}$ & $\mathbf{4 . 2 9 0 6}$ & $\mathbf{8 . 1 7 0 0}$ \\
Summer & $\mathbf{4 . 0 9 2 1}$ & $\mathbf{5 . 9 6 8 2}$ & $\mathbf{5 . 6 1 1 5}$ & $\mathbf{8 . 6 8 3 0}$ \\
Fall & $\mathbf{3 . 7 9 2 7}$ & $\mathbf{8 . 7 5 3 9}$ & $\mathbf{5 . 3 5 9 8}$ & $\mathbf{1 0 . 2 8 6 1}$ \\
\hline
\end{tabular}




\section{Conclusion}

In this paper various order TSK fuzzy systems were employed to forecast day ahead electricity price according to PJM market data. Tuning of these TSK fuzzy systems was done by using the Interior Point Method such that the proposed system gives the right output for an specific input. Results show the advantage of TSK fuzzy systems in forecasting electricity price in comparison with RBF neural network. However it was also seen that zero order TSK fuzzy system had the best performance among other TSK systems.

\section{References}

[1] Conejoa A.J., J. Contrerasa, R. Espi'nolaa and M.A. Plazasb, 2005. Forecasting electricity prices for a day-ahead pool-based electric energy market, International Journal of Forecasting, Vol. 21, No.3, pp.435- 462 .

[2] Raquel G., L.M. Romeo and A. Gil, 2006. Forecasting of electricity prices with neural networks, Energy Conversion and Management, Vol. 47, No.13-14, pp. 1770-1778.

[3] Li G., C.C. Liu, C. Mattson and J. Lawarrée, 2007. Day-Ahead Electricity Price Forecasting in a Grid Environment, IEEE Transactions on Power Systems, Vol. 22, No. 1, pp. 266-274.

[4] Garcia R.C., J. Contreras, M. van Akkeren, J. Batista, C. Garcia, 2005. A GARCH Forecasting Model to Predict Day-Ahead Electricity Prices, IEEE Transactions on Power Systems, Vol. 20, No.2, pp. 867-874.

[5] Hong Y.Y. and C.F. Lee, 2005. A neuro-fuzzy price forecasting approach in deregulated electricity markets, Electric Power Systems Research, Vol. 73, No. 2, pp. 151-157.

[6] Antonio J.C., M.A. Plazas, R. Espínola, and A.B. Molina, 2005. Day-Ahead Electricity Price Forecasting Using the Wavelet Transform and ARIMA Models, IEEE Transactions on Power Systems, Vol. 20, No. 2, pp. 1035-1042.

[7] Diego J.P. and J.R. Trapero, 2006. Electricity Prices Forecasting by Automatic Dynamic Harmonic Regression Models, Journal of Energy Conversion and Management, Vol. 48, No.5, pp. 1710-1719.

[8] Amjady N., 2006. Day-Ahead Price Forecasting of Electricity Markets by a New Fuzzy Neural Network, IEEE Transactions on Power Systems, Vol 21, No. 2, pp. 887-896.

[9] Nogales F.J., J. Contreras, A.J. Conejo and R. Espínola , 2002. Forecasting Next-Day Electricity Prices by Time Series Models, IEEE Transactions on Power Systems, Vol. 17, No.2, pp. 342-348.

[10] L.-X. Wang, A Course In Fuzzy Systems And Control, Prentice-Hall, Inc. Upper Saddle River, NJ, USA, 1997.

[11] J. J. Buckley, Universal fuzzy controllers, Automatica Vol. 28 Issue 6 (1992) Pages 1245-1248.

[12] R. A. Waltz, J. L. Morales, J. Nocedal, D. Orban, An interior algorithm for nonlinear optimization that combines line search and trust region steps, Mathematical Programming vol. 107 No. 3 (2006) pp. $391-408$. 
[13] Radial Basis Function :: Radial Basis Networks (Neural Network Toolbox), MATLAB 7.1, 2006 .

[14] Whei-Min Lin, Hong-Jey Gow, Ming-Tang Tsai, "Electricity price forecasting using Enhanced Probability Neural Network" Energy Conversion and Management 51, pp 2707-2714, 2010 